\title{
La conscience-avec comme événement-perceptif
}

\section{Consciousness-with as a perceptual event}

Ana Rita de Lima Ramos ${ }^{1}$

Recebido em $1^{\circ}$ de abril e aprovado em 16 de maio de 2019.

Résumé: Cet article prend comme point de départ une expérience médiatique immersive afin de penser toute expérience à partir d'une épistémologie relationnelle. En s'attardant à la relation comme dimension autonome, il propose de penser l'imbrication du virtuel et de l'actuel dans l'expérience de sorte à développer un modèle diagrammatique de la communication. L'expérience immersive sert de tremplin pour la pensée. Elle permet de sortir d'une dimension représentative de l'expérience et d'affirmer toute expérience comme acte de communication à son stade le plus pur. Ce faisant, l'article rend compte de la dimension affective de la communication en affirmant sa complexité à partir de la mise en oeuvre des concepts mode d'expérience, mode d'expression et mode d'existence. La relation devient ainsi angle d'analyse pour l'étude du corps comme plan d'émergence de qualités esthétiques. En d'autres mots, ce que qui est proposé est l'opportunité de penser la communication à partir d'une dimension intensive - celle de l'affect.

Mots-clés: Relation virtuel. Expérience. Événement. Qualité esthétique. Affect. Communication

\begin{abstract}
This article takes an immersive media experience as its starting point in order to think every experience through a relational epistemology. By dwelling on relation as an autonomous dimension, its purpose is to think of the imbrications of the virtual and the actual in experience so as to develop a diagrammatic model of communication. Here, immersive experience serves as a springboard for thought. It allows a departure from a representative dimension of experience and the affirmation of every experience as an act of communication at its purest state. In doing so, the article gives an account of the affective dimension of communication by asserting its complexity through the interweaving of the concepts mode of experience, mode of expression and mode of existence. Relation hence becomes the angle of analysis for the study of the body as a plane of emergence of aesthetic qualities. In other words, what is proposed is the opportunity to think communication from an intensive dimension - that of affect.
\end{abstract}

Key-words: Relation. Virtual. Experience. Event. Aesthetic quality. Affect. Communication.

Il faudrait que l'individu se saisisse lui-même comme événement. Et que, l'événement qui s'effectue en lui, il le saisisse aussi bien comme un autre individu greffé sur lui. Alors, cet événement, il ne le comprendrait pas, ne le voudrait pas, ne le représenterait pas sans comprendre et vouloir aussi tous les autres événements comme individus, sans représenter tous les autres individus comme événements (Deleuze, 1969 : 246). 
Le 4 mai 2012, la Société des arts technologiques de Montréal (SAT) exhibait dans son théâtre immersif appelé la Satosphère l'œuvre Hemisphere de ULF Langheinrich. La spécificité de ce dôme de $18 \mathrm{~m}$ de diamètre par $15 \mathrm{~m}$ de hauteur est la haute technologie investie dans l'art du son et de l'image permettant au spectateur d'être littéralement absorbé par l'ambiance créée au moyen des images projetées tout autour. En tirant profit de cette technologie, l'œuvre nous transportait à l'intérieur de son mode d'expérience propre. Sa forme signifiante (Langer, 1953), articulée à travers les textures sonores, rythmiques et visuelles (tous ces éléments qui jouent un rôle dans la constitution de l'œuvre comme symbole indivisible), s'est imposée à nous de façon indéniablement persistante. À la fin de la présentation, nous sommes restés un moment à contempler le mode affectif d'expérience qui nous traversait encore, complètement absorbés en lui, jusqu'à ce qu'une voix déclare: «vous pouvez maintenant rejoindre la réalité». Ces mots ont agi comme un seuil de passage d'un mode d'expérience vers un autre, régi par une différente structure affective. C'est-à-dire que ce qui s'est présenté à nous pendant le temps d'un visionnement avait une telle autonomie que nous en sommes sortis encore habités par son apparition. C'était tel un bouleversement sensoriel : les nuances de couleur et de son nous enveloppaient au-delà de toute tentative intellectuelle de comprendre ce qui se passait pendant la performance. L'œuvre sortait complètement de la dimension représentative et ne nous laissait que l'expérience en elle-même pour toute finalité.

Ce que la technologie actuelle permet de faire à travers une telle œuvre est de tracer des «territoires affectifs singuliers» à travers la mise en place de seuils perceptifs marqués. Une telle expérience esthétique est un événement, vécu pleinement dans l'activité du corps comme co-création d'une singularité. Cette dernière surgit comme l'irruption d'un élan de différenciation dans la qualité perceptive, ce que Whitehead appelle une tonalité affective (1933/1967). Selon cette approche événementielle de l'objet d'art médiatique, l'individu devient un champ ouvert à l'extérieur. C'est-à-dire qu'il y a interpénétration d'événements étant donné que le corps est immergé dans un entrecroisement relationnel qui est en soi un événement également. Ce faisant, la dualité sujet-objet est élargie à un complexe relationnel qui comprend autant l'humain que la technologie comme force agissante, entre autres. Ainsi, l'angle d'analyse ne se situe ni dans l'expérience subjective de l'individu, non plus dans un 
déterminisme technologique, mais dans la relation. Bien que cette dernière soit vécue sous forme de sensation, la tonalité affective émergeant du champ relationnel doit être comprise comme une valeur indépendante de celui qui la rencontre, même s'il participe à sa formation. Cette valeur indépendante est la qualité esthétique émergente : Suzanne Langer l'appelle sentiment objectif (1953). Ici, objectif sous-entend le fait que cette qualité est dotée d'une autonomie vis-à-vis de celui qui en fait l'expérience. Le rôle de l'individu dans l'expérience esthétique devient donc point de passage et d'activation d'une forme dynamique virtuelle qui s'actualise dans la forme signifiante.

La plupart des travaux concernant la technologie numérique, notamment en lien avec son pouvoir de créer des mondes immersifs, s'insèrent dans la perspective d'un spectateur subjectif devant une scène objective. Elle est naturelle si nous voulons comprendre le phénomène de la «réalité virtuelle», par exemple, qui nous présente la possibilité d'être complètement immergés dans un scénario créé numériquement, comme une réalité représentée. Sous-jacente à cette perspective est l'idée de la conscience conçue selon une dualité de l'expérience qui sépare l'individu des objets perceptuels. C'est ce que la langue allemande désigne par le mot Bewusstheit: une entité transcendantale, inchangeable et immuable. Cette notion de la conscience se réfère à un contenant que nous pouvons séparer de son contenu: l'expérience. Ce dualisme traite les faits de conscience et les faits matériels auxquels ils se rapportent comme deux segments différents parallèles. Dans ce cas, le spectateur regarde une scène extérieure (objective) à partir d'une intériorité (subjective). Seulement, contrairement à une peinture par exemple, dans le cas de l'expérience de l'art immersif, il n'y a plus la structure du cadre matériel pour séparer le corps de la scène. Dans l'épistémologie rationaliste, la fonction de la métaphore du «cadre» à travers lequel on regarde est d'assurer une certaine objectivité scientifique. Elle a cependant comme effet de séparer l'individu de ce qu'il observe et, sans la stabilisation d'un «cadre» extérieur, l'individu semble «déraciné», d'où l'impression d'irréalité. C'est là que la dualité qui sépare l'individu des objets perceptuels vacille. Le cadre d'une peinture est considéré comme étant objectif parce qu'il occupe (et, ce faisant, découpe) l'espace. Sans la séparation entre l'objet et le regard subjectif, il ne reste que le point de vue du sujet «censé» être séparé des objets de perception dont il fait l'expérience. 
Or, l'expérience de l'œuvre Hemisphere ne se délimite pas par un cadre, mais par le passage d'un seuil événementiel circonscrit par la durée pendant laquelle l'expérience prend place. Le cadre n'est même pas défini par les portes du dôme: bien avant que l'on y pénètre, les sons nous rejoignent, l'ambiance qui s'en dégage teinte l'expérience à venir - déjà en cours. Donc, ces éléments agissent sur l'expérience avant même que le dôme n'ait été pénétré. Ici, il ne s'agira pas de cadre, mais de seuil: le passage d'un seuil concerne la durée et le mouvement qu'elle exprime. Dans l'expérience Hemisphere, il n'y a pas de séparation spatiale possible: nous sommes l'expérience et elle nous englobe. En entrant dans le champ relationnel de son mode d'expérience, c'est le seuil d'un événement qui est franchi: nous pénétrons un champ d'expérience dans lequel se produit un engagement des sens dans une activité de collaboration ayant comme résultat l'avènement d'une valeur perceptive. L'expérience devient le terrain fertile à travers lequel des forces affectives déploient leur puissance. Ces forces font partie des facteurs formateurs de l'expérience esthétique au même titre que tout autre élément. Par conséquent, s'il n'y a plus de cadre pour séparer le sujet de l'objet, une vision spéculative du mode opérationnel du champ relationnel (créé par l'agencement entre affects, percepts, acteurs humains et technologies numériques) semble devenir une nécessité. Cette perspective fait partie d'un champ émergeant dans la discipline des études médiatiques. Il s'agit d'un angle d'analyse qui rompt entièrement avec une «rhétorique de dématérialisation» (Munster, 2006: 17) et qui renonce à une certaine apologie des médias pour se concentrer sur l'expérience comme événement.

En ce cas, nous affirmerons, comme William James, que la conscience n'existe pas (James, 2003: 2). En contrepartie, une compréhension du concept comme «fonction de connaissance» ${ }^{2}$ gagne en effectivité dans le cas d'une étude qui place le corps comme plan d'émergence de qualités esthétiques. En ce sens, il ne s'agira pas d'une «conscience de», car cela suppose une séparation, un regard extérieur aux choses. Si nous admettons que la conscience comme entité réceptive (comme contenant) n'existe pas, il ne nous restera alors que ce qui a été appelé le contenu de cette entité: l'expérience. ${ }^{3}$ Néanmoins, cette dernière ne doit pas être considérée seulement dans sa dimension d'appartenance à un individu spécifique - donc subjective -, mais avant tout comme relation: 
The first great pitfall from which such a radical standing by experience will save us is an artificial conception of the relations between knower and known. Throughout the history of philosophy the subject and its object have been treated as absolutely discontinuous entities; and thereupon the presence of the latter to the former, or the "apprehension" by the former of the latter, has assumed a paradoxical character which all sorts of theories had to be invented to overcome. Representative theories put a mental "representation," "image," or "content" into the gap, as a sort of intermediary. Common-sense theories left the gap untouched, declaring our mind able to clear it by self-transcending leap. Transcendentalist theories left it impossible to traverse by finite knowers, and brought an Absolute in to perform the saltatory act (James, 2003: 27-8).

Une telle proposition conceptuelle a l'avantage de prendre l'expérience comme ce qui se présente, tout simplement, dans un présent en train de se faire - une durée ${ }^{4}$. En ce sens, la relation est, en plus d'une rencontre, le mouvement qui génère cette rencontre liant sujet et objet comme une seule continuité. Telle que nous l'entendons, la relation n'est donc pas intersubjectivité , mais ce qui est «entre» l'objet et le sujet. En d'autres termes, ce qui est entre l'intersubjectivité ${ }^{5}$ et les complexes de configuration des objets. Pour James, la relation dépasse autant le sujet que l'objet. Elle se place en deçà d'un seuil d'indiscernabilité par rapport à l'expérience subjective ou objective. Sujet et objet n'ont pas une différenciation ontologique, mais de «fonction» - qui émerge à partir de la relation.

Il s'agit ici d'un geste de déplacement qui prend pour nœud central de problématisation non pas l'expérience subjective d'un sujet, mais bien les rencontres en soi comme «événement pur». Le mouvement d'émergence de la relation devient ainsi prioritaire par rapport au «positionnement» d'un sujet face à un objet d'expérience. ${ }^{6}$ Il n'y a donc pas attachement à une forme pré-constituée, mais bien à l'événement comme mouvement d'émergence constitutive. Ce qui se constitue à travers ces rencontres implique deux facteurs imbriqués l'un dans l'autre: de multiplicité et de mode. Le premier facteur nous ramène au fait que l'événement jaillit d'une multiplicité chaotique qui s'autoorganise sous la pression et par rapport aux limites des conditions héritées: le virtuel en voie d'actualisation. Le deuxième indique que l'avènement de cette constitution se fait d'une façon «singulière» qui lui est propre. ${ }^{7}$ La manière dont la multiplicité entre en rapport constitue l'individualité de la relation comme avènement d'un mode d'existence- 
qui, pour sa part, donne lieu à un mode d'expérience qui lui est propre. Le gain significatif qui s'accomplit avec cette inversion est l'accès à une dimension qualitative de l'expérience faite de potentiel et de puissance de transformation qui génère une «création continue d'imprévisible nouveauté» (Bergson, 1934: 99). Cette dernière se fait à partir de la rencontre entre les facteurs formateurs de l'expérience qui, conjointement, font émerger une qualité comme réalité relationnelle vécue - la qualité esthétique.

La plus grande difficulté avec ce schéma de pensée est qu'il met de l'avant «l'autonomie» non pas de l'individu ${ }^{8}$, mais de l'affect tel qu'il agit à l'intérieur d'un champ relationnel. La dimension de l'affect est faite de tendances, de densités et d'intensités qui se répartissent sous forme d'agencements dans un champ de potentiel qui ne contient pas de sujet ou d'objet, mais qui les produit. Tel que défini dans The Affect Theory Reader:

\begin{abstract}
Affect arises in the midst of in-between-ness: in the capacities to act and to be acted upon. Affect is an impingement or extrusion of a momentary or sometimes more sustained state of relation as well as the passage (and the duration of passage) of forces or intensities. That is, affect is found in those intensities that pass body to body (human, nonhuman, part-body, and otherwise), in those resonances that circulate about, between, and sometimes stick to bodies and worlds, and in the very passages or variations between these intensities and resonances themselves (Gregg et Seigworth, 2010:1).
\end{abstract}

La caractéristique de l'affect comme capacité d'agir et d'être agi, tel que défini par Gregg et Seigworth, souligne la vitalité qui lui est associée. Ainsi compris, il devient un ensemble complexe de rapports affectifs se constituant comme force qui passe à travers l'individu, malgré lui, et qui s'exprime comme durée qualitative. Aussi, non seulement l'affect est un événement qui passe à travers le corps, mais lui-même se constitue selon une certaine mobilité «entre» les facteurs formateurs de sa propre multiplicité. C'est par ce dynamisme qu'il se forme en tant qu'individualité et qu'il contribue, en tant que mode d'expression, à l'avènement d'un mode d'existence. En d'autres termes, alors que l'affect devient, il englobe et génère un mode d'expérience. Par exemple, toute joie peut contenir un certain niveau de tristesse, de sérénité, ou autres. La façon spécifique dont ces 
tendances s'agencent, se densifient ou s'alignent comme intensités génère la particularité de la qualité esthétique. Bref, cette durée est fondamentalement vécue selon le dynamisme et la complexité propres à l'agencement de l'affect en action, c'est-à-dire, sa singularité. Même avant que ces émotions ne soient reconnues par l'individu en tant que tel, l'affect agit déjà comme événement complexe (la sensation) au niveau de la conscience-affective. C'est-à-dire que l'affect possède une individualité qui lui est propre et qu'il la perfectionne à travers sa participation comme force relationnelle productrice de nouveauté.

Ce jaillissement continu d'actualité est l'assise même de la réalité au sens le plus général du terme et la dimension de laquelle il émerge porte un nom déjà bien connu: le virtuel. Or, il existe une polysémie du terme dans le champ des études en communication qui nous oblige à faire un survol de ses acceptions les plus connues. Breton et Proulx $(2006)^{9}$ divisent les études sur le virtuel dans le domaine des communications en trois lignes de pensée. Commençons par ce qu'ils appellent «la virtualité comme résolution». Lorsque les possibilités technologiques viennent répondre à la vie de tous les jours de manière créative et innovatrice, le «virtuel» devient synonyme de simulation informatique pour offrir un moyen d'explorer, d'amplifier et de démultiplier la réalité. Dans le même ordre d'idées, le terme peut aussi servir de tremplin pour signifier toutes les activités de réseautage social, ces dernières pouvant être perçues comme un affranchissement des contraintes de la matière, de l'espace et du temps (Breton et Proulx, 2006: 298). Nous sommes ici dans une perception résolument optimiste des possibilités technologiques.

La deuxième ligne de pensée saisit le virtuel comme «subordonné au réel», car ce dernier se comprend comme une re-présentation du réel, produite par un logiciel, dont l'existence n'a de substance que dans la perception humaine (Jones, 2005: 367) ${ }^{10}$ (Mais cette perception ne serait-elle pas déjà une réalité?). Dans cette perspective dualiste de l'expérience, il s'agit de la perception d'une réalité racontée, représentée, qu'on observe et à laquelle on ne prend pas vraiment part ou à peine. Dans «Envisioning the Virtual», Brian Massumi commente à cet effet:

The word "virtual" came into everyday use in the 1990s, as a rider on "reality." The rider overrode: the connotation was unreality. In the phrase "virtual reality," the adjective virtual stood as a synonym for artificial. Artificial, in this context, meant illusionary. The context, 
of course, was the dramatic registering in the popular imaginary that enormous changes were on the horizon with the dawning of the digital age. The first tentative steps toward the construction of interactive immersive environments had triggered hyperbolic worries--or hopes--that the fabled "cyberspace" of 1980s futurist fiction was on its way to supplanting "actual" reality. ${ }^{11}$

La perception produite numériquement étant ainsi considérée comme une réalité artificielle, elle est, par conséquent, analysée en termes de «réalité divorcée du monde» (Slouka, 1995) devenant un «simulacre», un «double» du réel (Baudrillard, 1981). Seulement, qu'arriverait-il si nous considérions la réalité virtuelle comme phénomène d'expression de la réalité, tel que le suggère Andrew Murphie (2002) ${ }^{12}$ ? Qu'arriverait-il si nous l'analysions à l'aide de la troisième approche pour laquelle le virtuel consiste en une dimension immanente au réel? En fait, le virtuel, tel que nous l'entendons, est rattaché à cet héritage philosophique qui, de nos jours, a été largement répandu par la pensée de Gilles Deleuze pour qui tout ce qui s'actualise est «en interrelation circulaire et productive» (Breton et Proulx, 2006:298) avec le virtuel.

\section{Sur la communication - et le virtuel}

Compte tenu de la relationalité qui lui est intrinsèque, le corps doit être compris comme une conscience-avec inclusive et activement participative dans le processus d'avènement du sens et de la valeur perceptive. La conscience-avec n'est pas l'unique responsable du sens, mais participe à sa constitution. ${ }^{13} \mathrm{Ce}$ qui donne lieu à une pensée processuelle de l'individu, qui se constitue à travers les expériences formatrices de son unité et individualité. Il s'ensuit que l'acte même de percevoir s'impose comme mouvement à travers lequel il y a production du monde parallèlement à une expérience directe de nous-mêmes: ce qui suggère une «action» (Munster, 2013:5). Celle-ci est opération à travers laquelle l'individu est constamment en train d'activer sa relation au monde. Ainsi, l'acte de perception prend place comme participation active à la relationalité émergente. Selon Simondon, la perception est «l'acte par lequel un sujet invente une forme et modifie par là sa structure propre en même temps que celle de l'objet: on ne perçoit qu'à l'intérieur d'un système tendu dont on est un sous-ensemble» (Combes, 1999 : 48). La «façon» dont cela se passe produit le sujet de la relation - qui ne doit pas être compris comme le sujet 
individuel qui effectue l'acte de percevoir. Il s'agit de la forme subjective (Whitehead, 1978): elle traverse le corps comme événement. Au bout du processus de communication, nous avons l'avènement du sens comme valeur perceptive vécue. Si nous remontons ce processus vers son émetteur, qu'est-ce qui communique? La cause est diffuse et distribuée. L'expérience est l'agencement par lequel s'effectue un bouillonnement d'activité entre une multiplicité de tendances, de densités et d'intensités. De ce processus, émerge la perception de la forme subjective. Ce qui communique, à travers l'expérience, est le potentiel réel (Whitehead, 1978) de la situation. Ce dernier doit être compris comme tout ce qui constitue virtuellement une situation particulière, la conditionnant de façon à forger son caractère différentiel. Le potentiel réel est, en ce sens, ce qui tranche, dans l'indétermination, la singularité d'une situation donnée. Cette singularité s'exprime comme forme subjective. Ce n'est pas d'une communication intentionnelle qu'il s'agit, mais d'une émergence spontanée de différenciation - ce jaillissement en lui-même est acte de communication à son stade le plus pur.

Ce qui se passe dans l'acte de perception est l'expérience vécue d'un «mode d'existence» englobant l'individu affectivement comme «mode d'expression». Par ce fait même, la forme subjective s'exprime et s'actualise, son apparition pouvant être reconnue par le fait que quelque chose de significatif survient. Ce que l'individu expérimente est l'actualisation d'un devenir qui se greffe momentanément au sien comme tonalité affective. Cette dernière est vécue comme qualité esthétique. C'est ainsi que la signification et le sens ne dépendent pas de l'individu qui reçoit/interprète/comprend un message, mais sont en soi un événement vécu. Dans ces conditions, l'expérience doit être considérée affectivement plutôt que cognitivement. Elle est une multiplicité qui s'actualise dans l'événement-perceptif par le mouvement d'agencement d'éléments virtuels qui se rencontrent et qui «tiennent ensemble» dans un «territoire» appelé l'expérience. ${ }^{14} \mathrm{Si}$ nous prenons cette dernière pour un territoire, le seuil perceptif sera la frontière entre territoires. Comme n'importe quelle frontière, le seuil n'est pas défini de façon stricte, mais il est un mouvement vers: un point de passage. Dans le flux continuel de notre devenir, notre «champ d'attention» (Bergson, 1934:169) passe d'un état affectif à l'autre. Il n'a pour seule réalité que l'immédiateté de l'instant actuel dans laquelle la conscience-avec 
participe à l'actualisation d'un ou de plusieurs modes d'existence. ${ }^{15}$ Ces derniers forment avec elle un ensemble-conscient qui, dans la relationalité émergente, génère un mode d'expérience. Ce qui catalyse cette relationalité est la singularité de ce qui émerge comme qualité esthétique. Donc, les seuils sont des points de passage d'un « mode d'expérience » à l'autre; leur fonctionnement est purement affectif, même s'ils agissent sur toutes les dimensions de l'expérience à la fois.

En somme, de la nature du corps humain, nous pouvons affirmer qu'il s'agit d'un point de passage de forces virtuelles qui, ce faisant, s'actualisent. C'est-à-dire que nous traiterons de la nature de l'expérience, de sa structure et de ses traces toujours en lien avec ces deux dimensions de la réalité: le virtuel et l'actuel. Attendu qu'il n'y a pas de sujet a priori, par conséquent, ce n'est que postérieurement à l'expérience que nous pouvons situer les catégories «sujet» et «objet» dans la relation. L'objet d'analyse devient, en ce cas, le processus d'actualisation du virtuel lui-même. Plus spécifiquement, il s'agira du devenir de la forme subjective. Ce qui assure l'objectivité scientifique dans cette épistémologie relationnelle est la nature ontologique de la relation. Lorsque James se réfère à l'expérience pure comme assise de la réalité, il sous-entend, par ce fait même, la possibilité, même infime, d'une perception directe de cette réalité virtuelle de l'affect. ${ }^{16}$ La difficulté d'une perception directe de l'expérience est conséquence du fait que, lors de l'actualisation, elle se situe à mi-chemin entre une virtualité pleine de potentialité réelle et l'actualité qui réalise son potentiel. Fuyante, elle est encore un futur antérieur. Néanmoins, malgré la difficulté d'une perception directe de l'expérience pure, elle laisse des traces affectives dans l'expérience.

Le fait que nous nous attardions justement à la relation comme dimension autonome (et non pas à l'appropriation de l'expérience comme subjectivité d'un sujet qui lui est préexistant) nous amène directement vers une vision non-anthropocentriste. Les conséquences d'un tel positionnement sont considérables compte tenu du fait que cela sous-entend une complexité inouïe. Que pouvons-nous attendre d'un tel champ de problématisation qui pose la relation avant l'individu, et qui le place comme participation (une conscienceavec) à un agencement dont l'expérience est un événement qui se vit comme émergence d'affect? La première conséquence est de pouvoir penser l'acte même de communication 
comme une pluralité de voix (autant que de voies) qui s'entrecroisent. Ce faisant, nous y ajoutons du bruit sous forme de forces affectives agissantes. Deuxièmement, le message devient une expérience vécue dont la base est purement affective: il est événement (Deleuze, 1969). Troisièmement, lorsque nous pensons l'expérience en lien avec les dimensions du virtuel et de l'actuel simultanément, nous venons de compliquer (ou plutôt d'intensifier) la recherche en communication.

C'est-à-dire que chaque expérience compte autant une dimension intensive qu'extensive. Si l'expérience pouvait être visualisée selon un diagramme, nous voudrions placer la durée chronologique sur un axe linéaire horizontal marquant la dimension extensive de l'expérience. Il s'agit de la durée telle que nous nous l'approprions: elle a un début, un milieu, une fin. Par contre, l'expérience n'est pas seulement cela. L'expérience contient un excès impossible à analyser réflexivement. Cet excès se présente comme l'intensité de l'expérience: toute une multiplicité qui dépasse la capacité réflexive de l'appréhender comme globalité. Ce débordement est une surcharge de potentialité. Cet excès, Ronald Bogue commente tel que suit:

Immanent within the active forces of bodies in formation, then, is a passive force of the virtual. Put another way, the actualization of the virtual must be described twice, once in terms of actual bodies and the standard physics of material forces, and a second time in terms of the passive syntheses of retentive contractions that make up the condition of possibility of all bondings and connections. The actualization of the virtual is a single process, but the passage of the virtual into the actual does not exhaust the virtual; the virtual remains immanent within the actual, an excess always in reserve, and that virtual immanent within the actual is manifest as sensation (Bogue, 2003:183).

Bogue appelle la manifestation de cet excès dans l'expérience la sensation. C'est une empreinte affective de la multiplicité indivisible composant cet excès d'information. Ce processus se fait par contraction des synthèses passives qui peuplent la dimension intensive de l'expérience. Selon cet auteur, il faut décrire l'actualisation du virtuel deux fois. D'abord, en termes du corps et de son système extensif, soit le monde objectif dans lequel il projette ses actions. Ensuite, en termes de synthèses passives. 
Si l'expérience comprend, en plus de la dimension extensive, cette dimension intensive, afin de mieux la saisir, il faut la comprendre en deux temps - bien que, dans l'expérience, il n'y ait pas une distinction de temps marquée. Ces deux actions parallèles assurent la collaboration circulaire constante du virtuel et de l'actuel. Premièrement, l'actuel du virtuel comprend tout ce qui est virtuellement en processus d'actualisation dans l'expérience. Deuxièmement, le virtuel de l'actuel, s'adresse à tout ce qui, déjà actuel, est en processus de virtualisation. Si l'expérience pouvait être visualisée selon un diagramme, donc, l'instinct serait de placer l'intensité sur un axe vertical qui traverse le premier - chaque point sur la ligne horizontale contiendrait son lot d'intensité propre. Mais cette verticalité n'est pas encore l'analogie parfaite, car il ne s'agit pas tellement d'une ligne, mais plutôt d'un champ virtuel «rhizomatique» ${ }^{17}$. À son degré le plus virtuel, ce champ atteint le potentiel pur (Whitehead, 1978). Mais, de façon concrète, l'expérience puise à même le potentiel réel de la situation pour faire émerger, à travers le processus d'actualisation, l'expression de la forme subjective. Le mode d'expression de la forme subjective actualise l'affect en cours de sorte que l'expérience fait émerger un mode d'existence radicalement nouveau: la singularité. Cette singularité est vécue comme mode d'expérience. Toutes les forces affectives à l'œuvre dans ce processus s'agencent comme synthèses passives qui, contractés dans l'expérience, sont vécues comme qualité esthétique sous forme de sensation. Ce schéma de l'expérience va du niveau le plus virtuel au plus actuel en passant par divers niveaux de virtualité. La tonalité affective les traverse tous. Elle est la base de l'expérience, car c'est elle qui est héritée d'une expérience à l'autre et, dans la pulsation d'une expérience à l'autre, c'est toujours d'une nouvelle singularité qu'il s'agit. Ce passage effectue deux actions simultanées. L'actuel du virtuel fait émerger l'événement d'actualisation. Le virtuel de l'actuel contient virtuellement en retour tout ce qui est produit par l'expérience et qui la dépasse réflexivement. Cet excès affectif participe au prochain jaillissement d'actualité comme ajout au potentiel réel de la situation: c'est ainsi que le virtuel et l'actuel se nourrissent continuellement.

Particulièrement, le fait d'inclure cette dimension virtuelle - et donc de l'affect - dans le champ d'études en communication nous permet de sortir des dichotomies et de passer à une «pensée relationnelle» ${ }^{18}$. Par exemple, dans le cadre de cette pensée 
non dualiste, «les notions d'intérieur et d'extérieur ne témoignent pas d'une différence ontologique. Il n'y a pas plus de monde extérieur préexistant qu'il n'y a de monde intérieur constituant. ${ }^{19}{ }^{19}$ Ici, Bergson nous invite à unifier ces deux termes apparemment contraires dans l'expérience: «En toute rigueur, on ne devrait pas parler de "monde intérieur" puisque l'on peut rester extérieur à soi-même. De même, on ne devrait pas parler de "monde extérieur", mais d'une tendance qui produit le monde comme extérieur» (Lapoujade, 2010:66). Cette part de nous qui demeure, selon Bergson, inconnue (extérieure à nousmêmes) est la dimension virtuelle de notre propre corps. Dans toute expérience, c'est comme si le corps traversait un territoire affectif. Tel un train qui traverse un paysage. On ne peut retenir tous les éléments qui s'agencent sous forme de territoire. Pourtant, l'atmosphère reste en nous imprégnée. C'est pourquoi chaque élément a tout de même un effet sur nous et cette multiplicité, vécue affectivement par la conscience-affective (la dimension virtuelle du corps), génère toute une gamme de sensations ${ }^{20}$ unifiées dans la singularité de la relation, soit la qualité esthétique.

En revanche, simultanément, un autre domaine de l'expérience est déjà en devenir. Si la sensation s'enroule en elle-même dans la conscience-affective pour intensifier l'expérience, la perception tâtonne déjà vers un arrêt du mouvement continu de l'événement en vue d'une (ré)action du corps ${ }^{21}$ La perception est donc directement liée au pouvoir d'action du corps - à son pouvoir extensif. Elle tend naturellement vers un positionnement face à ce qui se présente comme situation actuelle: l'émotion. ${ }^{22}$ Nous sommes maintenant en terrain connu: la conscience-réflexive implique l'activité symbolique de représentation sous forme distincte et séparée - la pensée. L'activité qu'elle génère est comme la pointe de l'iceberg en ce qui a trait aux complexités virtuelles de l'expérience : elle est directement affectée et constituée par la dimension affective qui est sa contrepartie et les deux sont constamment en collaboration dans l'acte de relation au monde. Autant la conscience-affective que la conscience-réflexive sont en soi des multiplicités qui génèrent des variations sur elles-mêmes en s'influençant différemment à chaque expérience. Dans la relation, elles se fondent en une seule et même expérience pure à seuils variables d'interpénétration. 
La conscience-réflexive nous permet de concentrer l'attention vers la compréhension et l'action. Mais elle n'est pas responsable de la préhension (Whitehead, 1978), car cette dernière consiste en la perception à son stade le plus pur - un état dans lequel il n'y a que la relation. L'expérience ainsi comprise englobe plusieurs dimensions du corps, de l'affection à son stade le plus pur jusqu'à l'activité qui s'insère dans le devenir en cours et qui s'étend sous forme de force d'affecter. Cet agencement ne peut pas être compris ou visualisé sous forme linéaire ni même selon une logique à arborescence. La structure la plus appropriée pour un modèle de communication qui tienne compte de la dimension virtuelle est la rhizomatique. C'est ainsi que le modèle de communication explose vers une vision diagrammatique de l'expérience. Cette dernière est diagrammatique, car elle opère par pure fonctionnalité d'action sur la matière:

Nous avons vu que les rapports de forces, ou de pouvoir, étaient microphysiques, stratégiques, multiponctuels, diffus, qu'ils déterminaient des singularités et constituaient des fonctions pures. Le diagramme ou la machine abstraite, c'est la carte des rapports de forces, carte de densité, d'intensité, qui procède par liaisons primaires nonlocalisables, et qui passe à chaque instant par tout point (Deleuze, 1986/2004:44).

C'est-à-dire que les forces virtuelles qui forment cette carte des rapports de forces s'agencent de manière à produire l'expérience sans toutefois la prédéfinir. Avoir une «vision diagrammatique» de l'expérience signifie comprendre cette dernière comme une réalité à part entière où l'individu agit en tant qu'élément formateur. Ainsi, on ne vit pas une expérience comme on subit des stimuli quelconques ayant un effet sur notre corps, mais on produit l'expérience conjointement avec des rapports de force sous le mode de l'agencement dans un champ relationnel englobant une multiplicité d'intensités.

Par conséquent, le terme «conscience-avec» place «le mouvement» comme force inhérente à toute forme d'agencement et son utilisation vient faciliter une compréhension de l'être comme devenir dans un devenir plus englobant duquel il fait partie: le monde. En saisissant l'expérience comme une multiplicité englobant plusieurs dimensions du corps, nous avons posé ce dernier comme «immédiatement autant abstrait que concret» 
(Massumi, 2002:31). Cette question de l'«immédiateté» indique deux faits parallèles. D'abord, le corps et l'environnement comme expression d'un seul et même devenir. Ensuite, elle indique l'idée de «simultanéité» de mouvements qui s'enracinent et qui se déploient à travers la relation. Ainsi, en ce qui a trait à la nature du corps, il s'ensuit que c'est autant avec notre corps qu'avec notre pensée que nous rencontrons le monde - d'où l'expression de l'événement-perceptif. Cette rencontre a lieu dans l'unification d'un complexe (l'expérience) qui englobe deux dimensions: le virtuel (exprimé dans la conscience-affective) et l'actuel (objectivé par la conscience-réflexive). Si nous partons de la relation comme réalité privilégiée et que nous posons les notions d'intérieur et d'extérieur en tant que «tendances», le corps et l'environnement deviennent dès lors deux parties «distinctes» d'un seul continuum. Un tel acte de la pensée vient briser l'opposition entre corps et pensée, entre corps et environnement. Il reste toutefois à préciser quelle est la nature de cette distinction, aussi bien que ses contours, notamment dans la façon comment individu et collectivité convergent dans la relation.

\section{Références}

BAUDRILLARD, Jean. Simulacres et simulation. Paris: Galilée, 1981.

BENNETT, Tony, Lawrence Grossberg, Meaghan Morris, and Raymond Williams. New Keywords: A Revised Vocabulary of Culture and Society. Malden, Mass.: Blackwell Pub., 2005.

BERGSON, Henri. La pensée et le mouvant: essais et conférences. Paris: F. Alcan, 1934.

BOGUE, Ronald. Deleuze on Music, Painting and the Arts. New York: Routledge, 2003. BRETON, Philippe, Serge Proulx, Philippe Breton, and Philippe Breton. L'explosion de la communication: introduction aux théories et aux pratiques de la communication. Paris: La Découverte, 2006.

COMBES, Muriel. Simondon individu et collectivité: pour une philosophie du transindividuel. Paris: Presses universitaires de France, 1999.

DELEUZE, Gilles. Foucault. Les Éditions de Minuit. Paris, n.d.

—. Logique Du Sens. Paris: Les Éditions de Minuit, 1969.

GREGG, Melissa, and Gregory J Seigworth. The Affect Theory Reader. North Carolina: Duke University Press, 2011. 
JAMES, William. Essays in Radical Empiricism. Mineola, N.Y: Dover Publications, 1912.

LANGER, Susanne K. Feeling and Form. New York: Charles Scribner's Sons, 1953.

LAPOUJADE, David. Puissances du temps: versions de Bergson. Paris: Minuit, 2010.

MASSUMI, Brian. A Shock to Thought: Expressions after Deleuze and Guattari. London; New York: Routledge, 2002.

Parables for the Virtual: Movement, Affect, Sensation. Durham, NC: Duke University Press, 2002.

MUNSTER, Anna. An Aesthesia of Networks: Conjunctive Experience in Art and Technology. Cambridge, Massachusetts: MIT Press, 2013.

- Materializing New Media: Embodiment in Information Aesthetics. Hanover, N.H.: Dartmouth College Press : University Press of New England, 2006.

SLOUKA, M. War of the Worlds: Cyberspace and the High-Tech Assault on Reality. New York: BasicBooks, 1995.

WHITEHEAD, A. N. Adventures of Ideas. New York: The Free Press, 1967.

Process and Reality. New York, NY: Free Press, 1978.

\section{Notas}

${ }^{1}$ PhD Comunicação, Concordia University, Montreal, Quebec, Canada. ana.ramos@umontreal.ca.

2 Cette «fonction», en allemand est désignée par le terme Bewusstsein, soit une compréhension de la conscience comme «mouvement-vers» l'acquisition de connaissance.

3 C'est-à-dire que l'expérience est la concrétisation, sous forme d'actualisation, d'un agencement de lignes virtuelles : un passage du virtuel à l'actuel. En ce sens, c'est un événement, manifestation d'un devenir qui prend déjà forme dans le domaine virtuel (fonction d'auto-organisation) et qui continue sont élan vers l'actualisation et au-delà. Conséquemment, l'expérience devient point de passage d'un devenir, entité autonome, affranchie, palpable - par la sensation: «Il y a là une rupture avec toute la tradition philosophique, qui mettrait plutôt la lumière du côté de l'esprit, et faisait de la conscience un faisceau lumineux qui tirait les choses de leur obscurité native. La phénoménologie participait encore pleinement de cette tradition antique; simplement, au lieu de faire de la lumière une lumière d'intérieur, elle l'ouvrait sur l'extérieur, un peu comme si l'intentionnalité de la conscience était le rayon d'une lampe électrique (« toute conscience est conscience de quelque chose...»). Pour Bergson, c'est tout le contraire. Ce sont les choses qui sont lumineuses par elles-mêmes, sans rien qui les éclaire: toute conscience est quelque chose, elle se confond avec la chose, c'est-à-dire avec l'image de lumière» (Deleuze, 1983:89). Ainsi, si pour la phénoménologie la conscience est cette lumière qui se pose sur les choses, dans la perspective ici avancée, l'événement en soi, l'expérience, est émergence de conscience et devient «la lumière».

${ }^{4}$ Que William James appelle le specious present (1950:609).

5 Tel que présenté, par exemple, par Alfred Schutz dans «Foundations of a Theory of Intersubjective Understanding» (1967:97-138).

${ }^{6}$ Pour une discussion sur la révolution bergsonienne en lien avec les notions de mouvement et de positionnement, voir Brian Massumi, «Concrete Is as Concrete Doesn’t» (2002:3-12).

${ }^{7}$ Ici, la «singularité » se réfère à ce qui échappe à une structure logique de catégorisation: c'est l'expression de nouveauté. Il ne s'agit donc pas d'un « particulier» comme cas qui entre dans une catégorie générale, mais de l'unique comme ce qui déborde toute forme de catégorie.

${ }^{8}$ Voir «The Autonomy of Affect » de Brian Massumi (2002:23-45).

9 Une brève introduction au sujet peut être trouvée dans Breton et Proulx dans «À la recherche du virtuel» 
(2006:296-299). La façon comment ces auteurs classent le terme vient largement inspirer la structuration de cette revue de littérature, sans toutefois que cette dernière s'y limite.

${ }^{10}$ Dans New Keywords: A Revised Vocabulary of Culture and Society publié par Bennett et al. sous «Virtual».

11 Dans The Oxford Handbook of Virtuality, ed. Mark Grimshaw (Oxford: Oxford UP, 2014), page 55.

12 Voir Andrew Murphie, «Putting the Virtual Back into VR», paru dans A Shock to Thought, 2002, édité par Brian Massumi.

13 Dans Nietzsche et la philosophie, Deleuze écrit à la page 5: «une chose a autant de sens qu'il y a de forces capables de s'en emparer. Mais la chose elle-même n'est pas neutre, et se trouve plus ou moins en affinité avec la force qui s'en empare actuellement.»

14 Dans Mille Plateaux, Deleuze et Guattari traitent de l'agencement territorial à partir de la ritournelle, l'élément musical étant traité surtout comme rythme. Voir pp. 381-433.

15 En d'autres termes, autant le corps que l'expérience sont des «territoires» dans le sens que le corps n'est jamais préconstitué, mais se définit et se constitue par l'ensemble-conscient qu'il forme avec les modes d'existence qui le traversent. Dans l'expérience, les territoires se superposent.

${ }^{16}$ Même s'il ajoute: «Only new-born babes, or men in semi-coma from sleep, drugs, illnesses, or blows, may be assumed to have an experience pure in the litteral sense of a that which is not yet any definite what, tho' ready to be all sorts of whats; full both of oneness and of manyness, but in respects that don't appear; changing throught, yet so confusedly that its phases interpenetrate and no points, either of distinction or of identity, can be caught. Pure experience in this state is but another name for feeling or sensation.» (James, 2003:49).

17 Deleuze et Guattari définissent les caractères principaux d'un rhizome dans l'introduction à Mille Plateaux, pp. 9-37.

${ }^{18}$ L'article de Didier Debaise «Qu'est-ce qu'une pensée relationnelle?» traverse la pensée de Simondon pour rendre compte d'une «véritable systématisation de la proposition "l'être est relation", la prise en compte explicite de ce qu'elle requiert pour pouvoir être posée et de ses conséquences dans différents domaines physique, biologique, social et technique.» Disponible en ligne http://multitudes.samizdat.net/Qu-est-ce-quune-pensee

19 David Lapoujade commente la pensée bergsonienne dans Puissances du temps, p. 66.

${ }^{20}$ Nous parlons de «sensation» tel que l'entend Bergson dans Matière et Mémoire, p. 52: «les sensations dont nous parlons ici ne sont pas des images perçues par nous hors de notre corps, mais plutôt des affections localisées dans notre corps même.»

${ }^{21}$ Encore une fois, référons-nous à Bergson tel qu'il définit la perception dans Matière et Mémoire, p. 57: «La perception, entendue comme nous l'entendons, mesure notre action possible sur les choses et par là, inversement, l'action possible des choses sur nous.»

22 Dans Parables for the Virtual, Brian Massumi définit: «An emotion is a subjective content, the sociolinguistic fixing of the quality of an experience which is from that point onward defined as personal. Emotion is qualified intensity, the conventional, consensual point of insertion of intensity into semantically and semiotically formed progressions, into narrativizable action-reaction circuits, into function and meaning. It is intensity owned and recognized» p. 28. 